\title{
Estudio bibliométrico del sistema de investigación local de San Luis Potosí (1920-2015)
}

\author{
Yoscelina I. Hernández-García* \\ Hugo Navarro-Contreras**
}

Artículo recibido:

9 de mayo de 2016

Artículo aceptado: 27 de septiembre de 2016

\section{RESUMEN}

Se presenta un estudio métrico de 1920 a 2015 de producción científica de instituciones del estado de San Luis Potosí. Se construye un instrumento de medición de la información a partir de dos bases de datos de corriente principal, una base de datos histórica y reservorios institucionales. El análisis final concentra 6,574 registros bibliográficos, firmados por autores pertenecientes a 59 instituciones potosinas. Las principales características encontradas en la literatura científica reflejan una alta concentración de colaboración científica y una clara relación entre las disciplinas cultivadas

Becaria posdoctoral PRODPD 2015, Universidad Autónoma de San Luis Potosi (UASLP).yosce2336@gmail.com, comhnavarro@uaslp.mx.

** Universidad Autónoma de San Luis Potosí (UASLP). hnavarro@uasIp.mx.

INVESTIGACIÓN BIBLIOTECOLÓGICA, Número Especial de Bibliometría, 2017, México, ISSN: 2448-8321. pp 249-270 
por instituciones potosinas y las áreas con potencial de desarrollo de innovación estatal.

Palabras clave: Bibliometría; San Luis Potosí; Producción científica; Sistema de investigación.

San Luis Potosí local science system's bibliometric study (1920-2015)

Yoscelina I. Hernández-García and Hugo Navarro-

Contreras

\section{Abstract}

An informational metric study of San Luis Potosí state's institutional scientific productivity from 1920 to 2015 is presented. The informational metric instrument is created from two mainstream data bases, a historic data base and institutional reservoirs. The final analysis was performed on 6,574 bibliographic records, signed by authors from 59 Potosinian scientific institutions. The main characteristics found in the scientific literature reflect a high concentration of scientific collaboration and a clear correlation between the scientific disciplines presented in Potosinian institutions and the estate areas of innovation and development.

Key Word: Bibliometrics, San Luis Potosí; Scientific productivity; Scientific system.

\section{INTRODUCCIÓN}

Ta comunicación científica es uno de los principales engranajes en la acti-

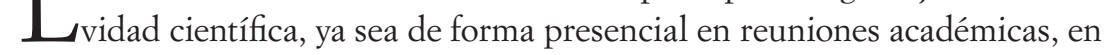
estancias sabáticas o en intercambios, los llamados colegios invisibles (Crane, 1972; Kretschmer, 1994), o en su forma más conocida a través de la literatura científica, conducen a la socialización de los resultados de la investigación, asegurando la construcción de un cuerpo de conocimientos comunes dentro de las comunidades científicas o incluso en su conformación.

En términos de medición de la información científica, son los artículos de este tipo los que permiten un espectro amplio de posibilidades en el estudio 
de tendencias y caracterizaciones de grupos de publicaciones, autores, instituciones, entre otros. Instrumentos como las bases de datos bibliográficas nos han permitido estandarizar la información que caracteriza la publicación y, por extensión, caracterizar autor(es) y su respectiva(s) institución(es), a la publicación (revista/journal), u otros elementos derivados de la información bibliográfica.

El proceso de comunicación científica se constituye a partir del filtrado y evaluación de los contenidos en la literatura científica, en donde el evaluador y el evaluado comparten credenciales e intereses, constituyendo un sistema de certidumbre en la comunidad científica (Garvey, 1972): "La Bibliometría, como disciplina instrumental de la bibliotecología, consiste en la aplicación de las matemáticas y los métodos estadísticos para analizar el curso de una determinada disciplina científica, así como a su comportamiento" (Chaviano, 2008), la medición de la información (como nombre genérico de las diferentes metrías) destaca la concentración, diseminación y uso de la literatura científica (Gorbea, 2005; Chaviano, 2008; Mutschke, 2015; Wolfram, 2015), para el estudio y evaluación de la actividad científica.

La variedad que se abre a los estudios de medición de la información se hace patente en las influencias de diferentes disciplinas como la sociología, psicología, historia, filosofía, por citar algunas; al igual que el enfoque y contextos en los que los estudios métricos forman parte, pero la constante que siempre se asoma es la de la evaluación para la toma de decisiones, ya sea para el descarte o selección de materiales en las unidades de información, o en la asignación de recursos a proyectos o investigadores, promoción en el escalafón institucional o en la elección de fuentes para publicar, tareas para las cuales las metrías de la información son una referencia importante.

En nuestro caso, hay un interés por observar el sistema de investigación local del estado de San Luis Potosí (SLP), caracterizando su producción científica institucional, y así obtener una imagen lo más completa y comprehensiva posible de su evolución y desarrollo, a través de sus resultados formales expresados como artículos científicos, que se registran en algún índice nacional o internacional.

En un sentido específico para Luna y colaboradores (2012), fue de interés la evolución e impacto de la Universidad Autónoma de San Luis Potosí (UASLP), dejando patente el periodo de consolidación que al momento de este estudio la institución estaba viviendo; en otro trabajo presentado en el Congreso Sinnco 2008, ${ }^{3}$ Moreno-Mata et al. (2008) realizaron una exploración del sistema de innovación del estado de San Luis Potosí, que se caracterizaba como 
incipiente. En un afán por acrecentar lo que hasta ahora se ha explorado sobre ciertos aspectos de la producción del estado de SLP, creemos que, si bien encontraremos coincidencias con los trabajos ya mencionados, la aplicación de un proceso más exhaustivo de normalización de las instituciones productoras de conocimiento aportarán detalles de las características de la entidad en su conjunto; esta visión global apunta a ser de utilidad tanto a los responsables de la toma de decisiones, como para quienes decidan continuar con la exploración de la producción estatal de SLP o cualquier otro estado de la república.

La originalidad de nuestro trabajo se sostiene en dos pilares, el primero es el método usado en la construcción de las herramientas de medición, en el que no se usa una sola fuente, sino que se reúnen cuatro diferentes bases de datos para así rastrear sesenta años de registros bibliográficos, balanceando la importancia de lo histórico con las tendencias más recientes, y permitiéndonos proyectar en dos etapas la investigación: la presente, en la que presentamos las tendencias generales, para posteriormente centrarnos en los aspectos de colaboración científica. ${ }^{4} \mathrm{El}$ segundo pilar de nuestro trabajo son las razones por las que se elige al estado de SLP, que tiene que ver con características del estado mismo así como de su sistema de investigación.

La situación geográfica de SLP es estratégicamente equidistante a la Ciudad de México, Guadalajara y Monterrey, las tres ciudades más grandes del país; además, cuenta con vías de comunicación férreas y carreteras importantes, lo que contribuye a que sea una de las economías más diversificadas de México (Conacyt, 2014). La importancia geográfica de SLP no sólo reside en sus colindancias, sino también en sus características internas: la entidad se divide en cuatro zonas (Huasteca, Región Media, Región Centro y Altiplano Potosino); en su ecosistema predominan los matorrales, bosques de encino y coníferas, pastizales y selvas secas y subhúmedas, dándole de igual forma una amplia variedad de fauna. Todas estas características nos plantean una latente relación con los intereses en temas de investigación o influencia en sus interacciones a través de su situación geográfica, dado que, de la misma forma en que es importantes su diversidad ecológica, hay una importante presencia en las zonas urbanas de industria en sectores como el automotriz, aeroespacial, industria alimentaria, minería, electrodomésticos, entre otras.

En cuanto al sistema de investigación estatal, se ha visto abiertamente apoyado desde los noventa, con la creación de un marco legal compuesto por el Consejo Potosino de Ciencia y Tecnología en 1996 (Copocyt), la promulgación de la Ley de Ciencia y Tecnología y la ley Orgánica del Copocyt en 2003. La aportación presupuestal estatal a las actividades de ciencia y tecnología 
son por debajo de la media nacional, ocupando el puesto 27 en 2012, mientras que el gasto privado respecto del pib estatal en el 2011 estaba por encima de la media nacional en el octavo lugar (Conacyt, 2014). El mismo Conacyt ubica a SLP en el cuarto lugar en aportación a la producción científica del país entre 2002 y 2012 (Conacyt, 2014: 64), mientras que el Foro Consultivo de Ciencia y Tecnología lo coloca en quinto lugar en el Ranking Nacional de Ciencia, Tecnología e Innovación del 2013 (FCCT, 2014).

Mientras existe suficiente información actualizada sobre la actividad científica, notamos un hueco en los aspectos históricos, lo cual nos interesa contribuir a analizar. La presentación de los resultados en este trabajo se divide en dos apartados: 1) se muestran las tendencias generales, 2) posteriormente se detallarán sus características bibliométricas a partir de instituciones pertenecientes al estado de SLP.

\section{Metodología}

Para la caracterización de la producción científica del estado de SLP, nos basamos en la metodología de Hernández et al. (2013), donde se destaca el uso de estrategias de búsqueda basadas en la dirección de adscripción, sobre la recuperación de grandes bloques de información, derivados de estrategias de búsqueda aplicadas a la fuente de publicación o categorías temáticas.

Dado que el objetivo del estudio es ser exhaustivo en el periodo de los conteos de producción científica, se usaron cuatro diferentes fuentes de recuperación ${ }^{5}$ de los registros bibliográficos para incluirlos en la base de datos bibliométrica (BDB); la fecha final del proceso de recuperación de las bases de datos bibliográficas fue 26 de junio de 2015. Los registros para la construcción de la BDB se obtuvieron de las bases de datos bibliográficas:

- Web of Science (WoS). Base de datos bibliográfica multidisciplinaria que indexa investigación de corriente principal, con una cobertura de aproximadamente de 11,500 revistas (Thomson Reuters, 2016). Se eligió este instrumento porque su esquema de selección de revistas a indexar asegura tener una base inicial selecta de registros bibliográficos. - Scielo (en la misma plataforma de WoS).

- Posteriormente se exploró la base de datos Scopus. Base de datos bibliográfica que indexa investigación de corriente principal, con una

5 Se lista el desglose de las fuentes de recuperación, pues se reconoce la distinción que en la base de datos bibliográfica Web of Science, se hace de los registros provenientes de Scielo, y ese mismo sentido reconocimiento a la producción que de la región de América Latina. 
cobertura aproximadamente de 21,500 revistas (Elsevier, 2016). Se eligió este instrumento como una forma de ampliar la visión de corriente principal de la producción científica de SLP.

- En tercer lugar, se eligió una base de datos histórica local del proyecto Atlas de la Ciencia Mexicana (ACM). Base de datos de uso exclusivo del grupo de Bibliometría Cinvestav-UNAM, cuyo objetivo es recolectar registros bibliográficos históricos sobre la ciencia en México. Se solicitó acceso a la información sobre el estado de SLP, para completar el aspecto retrospectivo de la investigación.

- Por último, se integró información del sitio web del Instituto de Investigación de Zonas Desérticas ${ }^{6}$ (IIZD) de la UASLP, siendo este instituto un importante referente histórico en la investigación local. ${ }^{7}$

También se exploraron, como una forma de control, las bases de datos siguientes:

- PubMed.

- INSPEC (en la plataforma de WoS).

- Los sitios de diferentes escuelas, facultades e institutos de la UASLP.

La información de todas las fuentes se integró en un solo bloque, y al eliminar los duplicados resultó que el $83 \%$ de la información bibliométrica identificada provenía de WoS, $12 \%$ de Scopus, $0.56 \%$ del sitio web de IIZD-UASLP, $0.37 \%$ de la BDB histórica ACM y el 3.6\% de Scielo.

Las fuentes utilizadas nos aseguran mantener una visión de corriente principal internacional en nuestros resultados, además de la aportación de la visión regional de Scielo, y la complementariedad de las fuentes históricas. Una vez que se incorporaron los registros bibliográficos en un manejador de bases de datos, se procedió a la normalización. Comúnmente ésta se centra en el campo de adscripción, pero en nuestro caso, al tener registros de diferentes procedencias, también se estandarizaron los nombres de las revistas, los tipos de documentos, y los idiomas de publicación.

Desde el inicio de la investigación, se planteó como uno de los objetivos principales la identificación de las instituciones de SLP que participan de la actividad científica, por lo que hubo especial cuidado en potenciar la información contenida en el campo de adscripción, del cual se derivaron dos niveles de normalización de la institución: para el primer nivel se incluyeron 
todas las instituciones; en el segundo, se trabajó a detalle: a) la UASLP, $b$ ) se desglosa dentro de las siglas SS (Secretaría de Salud) al "Hospital Central Dr. Ignacio Morones Prieto" y c) el Instituto Potosino de Investigación Científica y Tecnológica (IPICYT); en los tres casos por el importante papel que cumplen en la actividad científica del estado.

Además de la normalización de las instituciones propiamente dichas, también se identificaron los países de cada una de las adscripciones en los registros bibliográficos, esta identificación se hizo asignando el código de tres letras ISO 3166-1 alpha-3 para países. ${ }^{8}$ Para el caso del resto de los estados del país se designaron las abreviaturas oficiales para cada caso.

Por último, se usó la clasificación del Registro Nacional de Instituciones y Empresas Científicas y Tecnológicas (RENIECYT) para identificar todas las instituciones correspondientes al código de país MEX (México); sumando a esta clasificación un guión seguido de una letra "S", si la empresa o institución de la administración pública se orientaba a actividades de salud; un guión y la abreviatura "PRIV" si la institución de educación superior, era de carácter privado; también se incluyeron las siglas para la identificación de instituciones de educación media superior y de educación básica; dejando al final diez formas diferentes de identificar la actividad de cada una de las instituciones (véase la Figura 2).

\section{RESULTADOS Y DISCUSIÓN}

\section{Producción general}

El total de la base de datos está integrada por 6,574 trabajos publicados a lo largo de sesenta años de estudio de la producción científica de San Luis Potosí. La Figura 1 contrasta el total de producción de SLP, con los trabajos en colaboración ( $78 \%$ ) y los trabajos individuales (21\%), siendo muy significativa la participación de más de una institución en las publicaciones.

Cabe aclarar que más adelante se hace un conteo de las coautorías, es decir, cuando hablamos de colaboración nos referimos a los conteos a partir de la dirección de adscripción, y la coautoría la observamos en el campo de autor. 
Figura 1. Producción científica y tipos de participación institucional del sistema de investigación de San Luis Potosí (1920-2015)

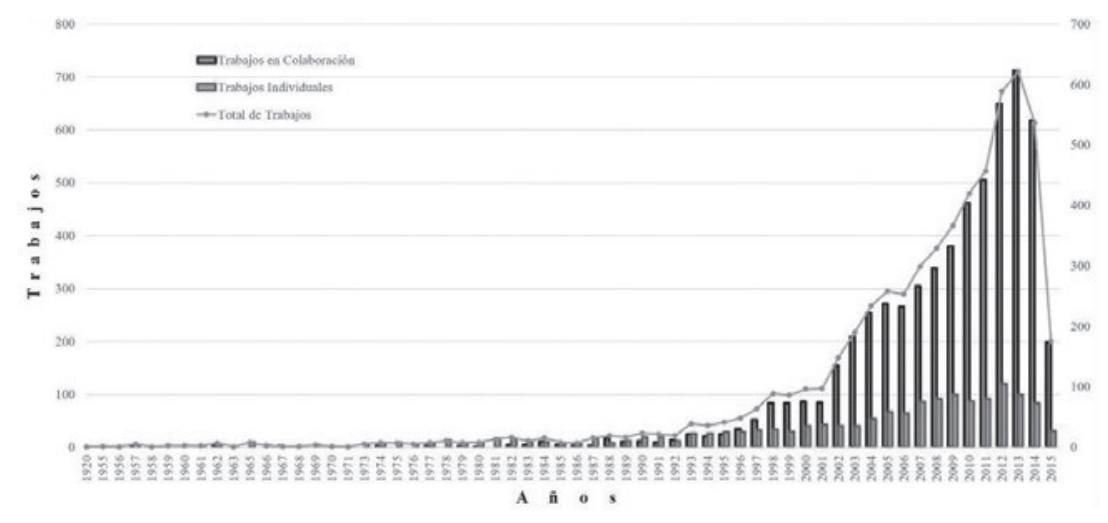

Fuente: elaboración propia.

Una de las reglas más importantes al momento de construir una base de datos bibliométrica es respetar la información que se extrae de las fuentes de información secundarias, por lo que en el caso de la producción científica de SLP, las seis décadas de estudio no son continuas, incluso el mismo periodo (1920-2015) se derivó de lo que arrojaron las bases.

El primer registro de una publicación científica es de 1920, donde se encuentra el primer trabajo con adscripción a SLP y de ahí no se vuelven a encontrar registros, reiniciándose hasta 1955. Posteriormente, hay otros dos años en los que no hay registro de publicaciones de instituciones potosinas (1964 y 1972).

La Figura 1 muestra un importante sesgo a la derecha de la gráfica, probando que la acumulación de esfuerzos durante las primeras décadas; iniciando en 1950, empieza a tener frutos hacia los últimos veinte años anteriores al momento actual, misma tendencia que se ha identificado en otros trabajos de medición de la información a las que han sido sujetas algunas de las instituciones de SLP; por ejemplo, Luna et al. (2012: 185) señalan que "la UASLP presenta a partir de 1994 un crecimiento estable, con ligeros incrementos en algunos años consecutivos, y obtiene una contribución porcentual en el impacto de la ciencia mexicana que aumentó del 0.26\% en 1980 al $0.99 \%$ en $2008 \%$.

Cabe señalar que los trabajos en colaboración son predominantes en los años finales del estudio. En las primeras décadas de la Figura 1 son más los trabajos individuales. Durante más de treinta años se privilegió la 
publicación individual a la de participación con otras instituciones, nacionales o extranjeras; $\mathrm{y}$ hacia los últimos años se ha adoptado un claro interés o necesidad de colaborar, posiblemente reflejando además la circunstancia de que los problemas a estudiar demandan técnicas o visiones que por necesidad aportan más de un solo individuo.

Aunque midamos de forma separada la colaboración institucional y la coautoría, es innegable la íntima relación entre estas formas de la producción científica; pero su composición sí tiene el potencial de mostrar formas de socialización dentro de las disciplinas, o en nuestro caso de un sistema local de investigación. Si nos encontramos una sola adscripción, no necesariamente tiene que traducirse en que está firmando un solo autor, puede listarse una amplia coautoría perteneciente a la misma institución. Los porcentajes respectivos son $95 \%$ en coautorías y $5 \%$ de individuales.

De igual forma, la colaboración representa una práctica frecuente; la coautoría es también importante para la producción científica de esta entidad. Dada la importancia de ambos conteos, aquí sólo se observarán de forma general y en trabajos posteriores se publicarán los detalles de ambas tendencias.

Si a la lectura de la Figura 1 se asocia la notable participación coautoral, se obtiene que la participación con instituciones fuera de SLP y de México son una característica predominante en los patrones de publicación de las instituciones potosinas, en donde la construcción de redes de colaboración cumplirían un importante papel en el posicionamiento del sistema de investigación del estado potosino.

Una de las incorporaciones del WoS a la descripción que hace de los registros bibliográficos es la identificación que los autores señalan de su pertenencia a algún grupo de colaboración; el Cuadro 1 muestra los nombres y conteo por periodos en los que investigadores potosinos han participado durante los últimos quince años, siendo ésta la información que se recupera con nuestras estrategias de búsqueda. 
Cuadro 1. Grupos de colaboración en la producción científica del estado de slp

\begin{tabular}{|l|l|c|c|c|c}
\hline \multicolumn{1}{|c|}{ Grupo de Colaboración } & $\mathbf{2 0 0 0 - 2 0 0 4}$ & $\mathbf{2 0 0 5 - 2 0 0 9}$ & $\mathbf{2 0 1 0 - 2 0 1 5}$ & Trabajos: \\
\hline 1 CMS Collaboration & 0 & 2 & 361 & 363 \\
\hline 2 ATLAS Collaboration & 0 & 0 & 81 & 81 \\
\hline 3 SELEX Collaboration & 11 & 2 & 0 & 13 \\
\hline 4 UPAMIGOS Grp & 0 & 0 & 5 & 5 \\
\hline 5 NA62 Collaboration & 0 & 0 & 3 & 3 \\
\hline 6FrPNC Collaboration & 0 & 0 & 2 & 2 \\
\hline 7PRINTO; PRCSG & 0 & 1 & 1 & 2 \\
\hline 8 APPRAISE-2 Investigators & 0 & 0 & 1 & 1 \\
\hline 9 ATLAS Collaboration; CMS Collaboration & 0 & 0 & 1 & 1 \\
\hline 10 AURORA Study Grp & 0 & 1 & 0 & 1 \\
\hline 11 BLISS-52 Study Grp; BLISS-76 Study Grp & 0 & 0 & 1 & 1 \\
\hline 12 CIBOMA GEICAM & 0 & 0 & 1 & 1 \\
\hline 13 CLARITY TIMI & 0 & 1 & 0 & 1 \\
\hline 14 CMS Collaboration; TOTEM Collaboration & 0 & 0 & 1 & 1 \\
\hline 15 HPV VVAP Study Grp & 0 & 0 & 1 & 1 \\
\hline 16 Registro Nacl Mexicano & 0 & 1 & 0 & 1 \\
\hline 17 Soc Iberoamer Neonatologia SIBEN & 0 & 0 & 1 & 1 \\
\hline 18 TRILOGY ACS Investigators & 0 & 0 & 1 & 1 \\
\hline Total & & & & $\mathbf{4 8 0}$ \\
\hline
\end{tabular}

Fuente: elaboración propia.

Uno de los procesos de colaboración más visibles en San Luis Potosí es la participación de investigadores del Instituto de Física de la UASLP en la investigación en física de altas energías (FAE), uniéndose a otras instituciones mexicanas en la gran ciencia (Collazo y Luna, 2002; Collazo et al., 2010).

Los grupos de colaboración en las posiciones 1, 2, 3, 5, 6, 9 y 14 que se muestran en el Cuadro 1 corresponden a investigaciones relacionadas con FAE, siendo las de publicaciones más numerosas y recientes, como se aprecia en la composición del Cuadro 1.

El resto de los once grupos de colaboración donde se han integrado investigadores de SLP tienen que ver con temas de Medicina: UPAMIGOS Grp investiga el tratamiento y factores de riesgo de la obesidad (infantil), especialmente relacionado con determinantes genéticos; PRINTO; PRCSG, es un grupo de investigación asociado a la Organización Internacional de Pruebas en Reumatología Pediátrica; APPRAISE-2 Investigators, aborda las pruebas clínicas en pacientes con Síndrome Coronario Agudo tratados con Apixaban; AURORA Study Grp, Evalua el uso de Rosuvastatin en pacientes que utilizan hemodialisis regular; BLISS-52 Study Grp; BLISS-76 Study Grp, realiza pruebas clínicas que evalúan la eficacia y seguridad del uso de Belimumab en pacientes con Lupus Eritematoso Sistémico; CIBOMA GEICAM, grupo de colaboración inscrito en el marco de la Coalición 
Iberoamericana de Oncología Mamaría; CLARITY TIMI 28 Investigators, desarrolla estudios sobre Clopidogrel como ayuda en temprana repercusión en Trombosis en infarto al miocardio; HPV VVAP Study Grp es una Red Mundial de estudios de atributos genotípicos en el papiloma humano; Registro Nacl Mexicano, es el grupo que trabaja en el Registro Nacional Mexicano de Enfermedad Vascular Cerebral; Soc Iberoamer Neonatologia SIBEN, son investigadores con la Sociedad Iberoamericana de Medicina y Enfermería Neonatal; y el grupo TRILOGY ACS Investigators, que trabaja en pruebas clínicas en el tratamiento del síndrome coronario agudo. Los años de las publicaciones de estos grupos de colaboración, una vez más demuestran la reciente consolidación de prácticas colaborativas, trátense de temas "big science", o de otras disciplinas.

Cabe señalar que Moreno et al. (2008) identifican al sector salud como una de las áreas prioritarias en materia de innovación, dadas sus características de consolidación en recursos humanos calificados, infraestructura institucional y potencial de investigación; con nuestros resultados se agrega la participación en la producción científica local, y una importante participación en grupos de colaboración en temas específicos y de interés internacional.

En total, nuestra base de datos incluye 1,666 diferentes títulos de revistas, 203 de los cuales se asocian a alguna categoría en Física, de entre otros títulos los que incluyen la FAE concentran el 10\% del total de registros bibliográficos (véase el anexo 2).

El Cuadro 2 se dividió en tres grandes periodos: iniciando con el año en el que se identifica la primera publicación de alguna institución de SLP, hasta los últimos quince años de mayor concentración de trabajos, pasando por lo que denominaríamos periodo de cimentación de las infraestructuras humanas y físicas para la ciencia en SLP, durante las décadas de los ochenta, noventa y primer lustro del $2000 .^{9}$ 
Cuadro 2. Revistas que concentran más artículos de instituciones potosinas, por periodo

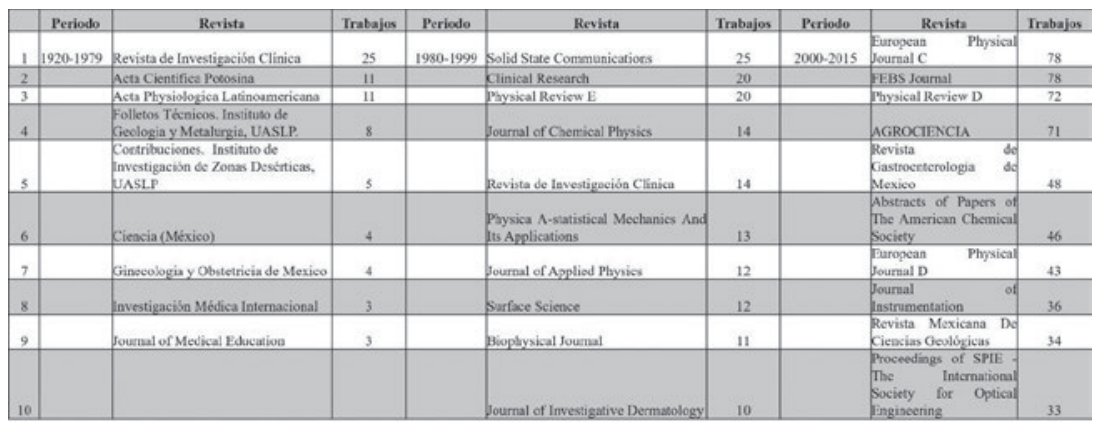

Fuente: elaboración propia.

Un comportamiento natural en la información científica, asociada a las leyes empíricas de la bibliometría, es la concentración de cierto porcentaje de documentos en unos pocos títulos de revistas (Gorbea, 1996; Archuby, 2006; Ram y Paliwal, 2014), por lo que para el acomodo y selección de títulos en el Cuadro 2 sólo se enumeran los títulos que integran mayor número de trabajos; y para efectos de comparación se tomó como base el segundo periodo, en el que los títulos de las revistas que publicaron menos de diez trabajos no son consignadas.

En el periodo de inicio de la investigación en SLP (1920-1979), se observan títulos de revistas de alcance más local, en temas de medicina, geología y botánica. Justamente en este periodo es cuando la mayoría de las escuelas y facultades de la UASLP se fundan. Es importante mencionar la influencia de la UASLP en la producción científica del estado, pues, como se verá más adelante, es una de las instituciones más importantes de la entidad. En total, en este periodo se identificaron 53 diferentes títulos de revistas.

En el periodo 1980-1999, que denominaremos como de cimentación, se contabilizaron 246 fuentes, de las cuales se muestran 10 en el Cuadro 2, el total de trabajos en el mismo periodo representa el $33 \%$, dejando una larga lista de revistas en las cuales, aunque dispersamente, también se publicó. En el último periodo del Cuadro 2 que se describiría como en proceso de consolidación, se muestran 10 de las 1,538 revistas, dejando ver la variedad de temas (medicina, química, física, geología y agrociencias) que se abordan a través de las disciplinas que se cultivan en las instituciones potosinas, tan sólo en la muestra de diez títulos se concentra el 14\%. 


\section{Producción InSTITUCIONAL}

Una vez completado el proceso de normalización, fue posible identificar 59 instituciones adscritas al sistema de investigación de SLP, a lo largo de seis décadas de estudio de su producción científica. El Cuadro 3 muestra las siglas asignadas a las instituciones potosinas, seguido de las décadas en las que se encontró alguna participación en publicaciones de corriente principal; al final se suman estas participaciones para cada caso.

Se habla de participaciones y no de documentos individuales, ya que, como se mostró antes, la producción científica de SLP tiene una alta participación de trabajos en colaboración, por lo que en un mismo registro bibliográfico, de los 6,574 que se recuperaron, puede contener más de una dirección de alguna institución potosina. Así pues, en la última columna de izquierda a derecha se cuenta el total de participaciones por institución, y en la última fila de nuevo se hace una sumatoria de participaciones, ahora por década analizada.

El orden dentro del Cuadro 3 es descendente a partir del número de participaciones de cada de las 59 instituciones, ${ }^{10}$ dejando a la UASLP $(6,056)$ en la primera línea, seguida del IPICYT (1,423), como las más visibles del sistema de investigación local.

Hay una importante participación de instituciones relacionadas con el sector salud, tanto del sector público como del privado; las de mayor participación en orden descendente a la magnitud de su producción científica acumulada publicada son la Secretaría de Salud (354), los centros de salud y hospitales pertenecientes al Instituto Mexicano del Seguro Social (79) y el Hospital Ángeles (53).

10 En el anexo 1, se puede consultar el listado de siglas y nombres desglosados de las instituciones y las dependencias en los Cuadros 4 y 5 . 
Cuadro 3. Producción científica institucional de San Luis Potosí (1920-2015)

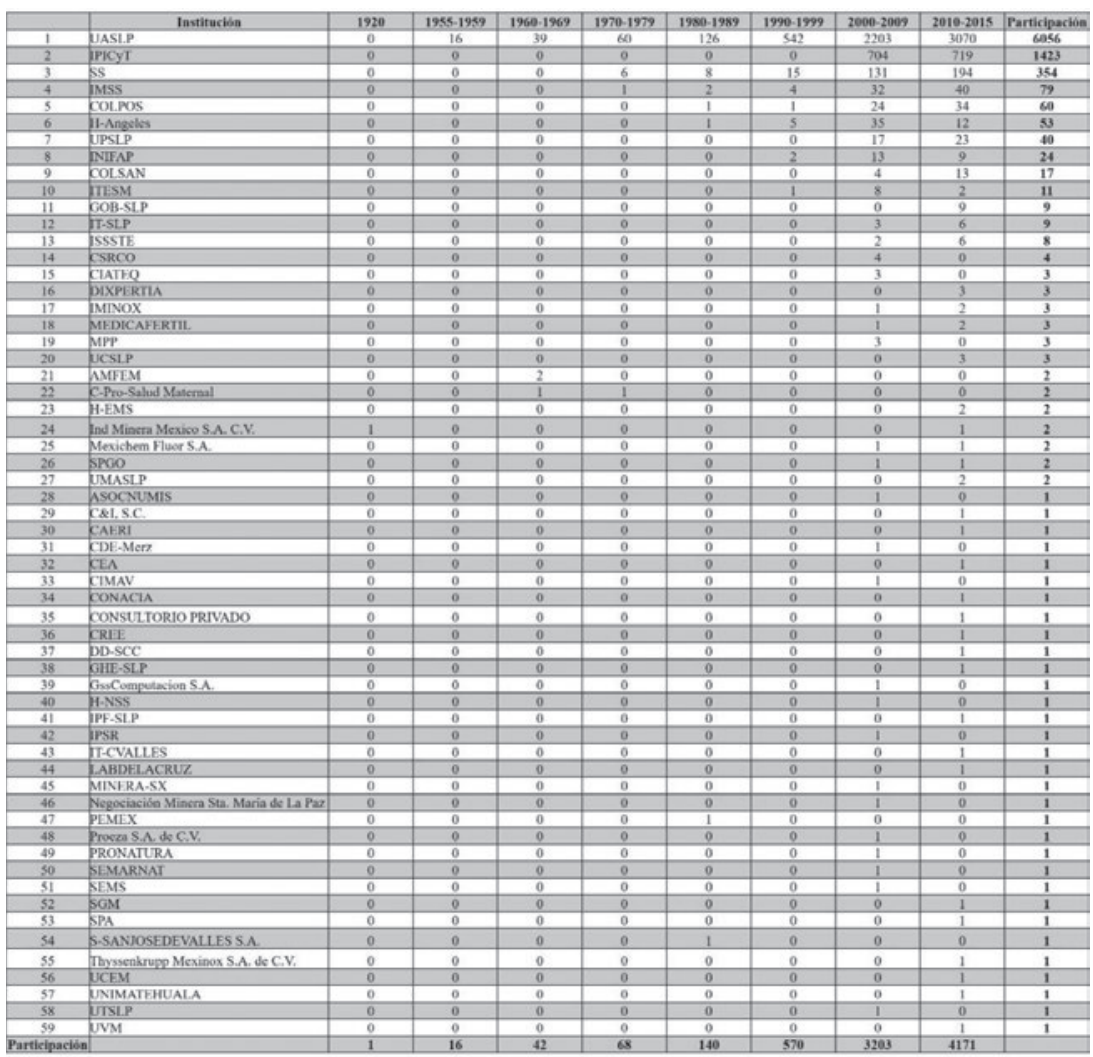

Fuente: elaboración propia.

Siendo uno de los principales objetivos de ese trabajo cubrir el mayor periodo de tiempo posible de la producción científica de SLP, en el Cuadro 3 se dividieron los sesenta años en siete columnas, consignándose en una columna adicional al año 1920, en la que se detectó la primera publicación de una institución potosina, de la empresa "Industrial Minera Mexico, S.A. de C.V." que, sin embargo, le toma otros sesenta años, según nuestro estudio, para participar nuevamente en la producción científica del estado, con un trabajo en colaboración internacional entre México-Argentina, en el que también participa la UASLP y se publica en la revista Surveys in Geophysics, mientras que la publicación de 1920 es un trabajo individual en el Transactions of the American Institute of Mining and Metallurgical Engineers.

Es común que, en los casos en los que se encuentra sólo una participación (Cuadro 3), sea un efecto del proceso de colaboración entre una de las 
instituciones líderes o de mayor producción, con instituciones con menos recursos materiales, humanos, informacionales o que están iniciando sus actividades de investigación; en el caso del sistema de investigación de SLP, el $51 \%$ de los casos entra en esta descripción.

SLP, desde principios del siglo XX, inició el desarrollo de la actividad industrial y junto con políticas que buscan acelerar este desarrollo, claramente permanentes desde los años cincuenta; algunos de los centros de investigación con vocación de apoyo y de promoción técnico-industrial-manufacturera también contribuyen a la producción científica, representados en las sedes domiciliadas en el estado, del Ciateq (posición 15 en el Cuadro 4) y el Cimav (posición 33 en el Cuadro 3).

Un caso aparte lo constituye El Colsan (quinta posición en Cuadro 3), ya que, por la naturaleza de su vocación científica, orientada hacia las ciencias sociales, sus hábitos de publicación no se consignan necesariamente en las bases de datos bibliométricas consideradas metodológicamente en este trabajo, y en general lo que aparece son conteos menores a sus totales reflejados en otro tipo de formatos, como el libro.

Como se menciona en el apartado de la metodología, se normaliza en segundo nivel, los casos de la UASLP, ss e IPICYT; siendo las tres instituciones líderes en la producción científica del estado de SLP. El Cuadro 4 desglosa las participaciones de las escuelas, facultades e institutos que integran la UASLP, las aportaciones de los cinco departamentos y dos de los laboratorios nacionales asociados al IPICYT; y las participaciones del Hospital Central "Dr. Ignacio Morones Prieto". 
Cuadro 4. Producción científica desglosada principales dependencias de Instituciones potosinas

\begin{tabular}{|c|c|c|c|c|c|c|c|c|c|c|c|}
\hline $\begin{array}{l}\text { Dependencias } \\
\text { UASIP. }\end{array}$ & Farticipación & $\begin{array}{l}\text { Periodo de } \\
\text { Productón } \\
\text { Clentifica }\end{array}$ & $\begin{array}{c}\text { Abo de } \\
\text { Fuadacion }\end{array}$ & $\begin{array}{c}\text { Dependenciss } \\
\text { IPICYT }\end{array}$ & Participacion & $\begin{array}{l}\text { Periodo de } \\
\text { Production } \\
\text { Cientificas }\end{array}$ & $\begin{array}{l}\text { Atho de } \\
\text { Fendaclón }\end{array}$ & Dependencia Ss & Participaclón & \begin{tabular}{|l} 
Periododo de \\
Produccion \\
Cientiflea
\end{tabular} & \begin{tabular}{|c|} 
Ako de \\
Fundacke
\end{tabular} \\
\hline & 1460 & $1981-2015$ & 1956 & DMav & 513 & $2000-2015$ & 2000 & $\begin{array}{c}\text { HC- } \\
\text { Dr-Mosonestricto }\end{array}$ & & $1977-2015$ & 1946 \\
\hline 2 FACMED & 1201 & 1957.2015 & 1923 & D8M & 312 & $2002 \cdot 2015$ & 2000 & & 318 & & \\
\hline $\begin{array}{l}3 \\
4 \\
4 A C C N G M \\
\end{array}$ & 727 & 1988.2015 & 1928 & bCA & 244 & $2002 \cdot 2015$ & 2000 & & & & \\
\hline \begin{tabular}{l|l}
4 & FACING \\
5 & ICO
\end{tabular} & $\frac{481}{436}$ & $1987-2015$ & 1945 & DMA & 235 & 200222015 & 2000 & & & & \\
\hline $\begin{array}{l}5 \\
6 \\
6\end{array}$ & $\begin{array}{l}436 \\
420\end{array}$ & $\frac{1990.2015}{1985-2015}$ & $\frac{1990}{1956}$ & $\begin{array}{l}\text { DGA } \\
\text { INAN }\end{array}$ & $\frac{74}{36}$ & $\frac{2002-2015}{2008-2015}$ & 20006 & & & & \\
\hline 7 IMETALUR & 285 & $1995-2015$ & 1960 & cNS & 9 & $2004-2011$ & 2006 & & & & \\
\hline 8 IIIZD & 234 & 1955.2015 & 1954 & & & & & & & & \\
\hline 9 FACESTOM & 165 & $1985-2015$ & 1946 & & & & & & & & \\
\hline 10 FAGV & 1115 & 1986.2015 & 1972 & & & & & & & & \\
\hline 11 CIACYT & 102 & $2005-2015$ & 2009 & & & & & & & & \\
\hline 12 IGEOL & 91 & 1985.2015 & 1987 & & & & & & & & \\
\hline 12 FACENF & 63 & $2005-2015$ & & & & & & & & & \\
\hline 3 COARA & 51 & $2008-2015$ & & & & & & & & & \\
\hline 4 PACPSI & 40 & $2004-2015$ & 1972 & & & & & & & & \\
\hline 15 FACCSH & 34 & $2006-2014$ & 2002 & & & & & & & & \\
\hline 6. PMPCA & 31 & $2007-2014$ & 2002 & & & & & & & & \\
\hline & 30 & $1974-2015$ & 1938 & & & & & & & & \\
\hline 18 UZM & 25 & 1998-2015 & 1984 & & & & & & & & \\
\hline 9 UZH & 19 & $2010-2015$ & 1984 & & & & & & & & \\
\hline 0 CEASSA & 8 & $\frac{2008-2013}{20.014}$ & & & & & & & & & \\
\hline 1 FACECON & 8 & $2005 \cdot 2014$ & 1964 & & & & & & & & \\
\hline $\begin{array}{l}2 \text { 2 FACHAB } \\
3 \text { FCI }\end{array}$ & $\frac{8}{7}$ & $\begin{array}{l}2003-2014 \\
2008.2013\end{array}$ & $\frac{1972}{1980}$ & & & & & & & & \\
\hline 4 AGENAMBI & 6 & $2006-2015$ & 1998 & & & & & & & & \\
\hline 5 ECC & 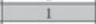 & 2000 & 1985 & & & & & & & & \\
\hline 6 CARIIS & 1 & 2014 & & & & & & & & & \\
\hline
\end{tabular}

Fuente: elaboración propia.

Conviene mencionar que en el Cuadro 4 los totales de las participaciones por dependencia en cada institución no necesariamente coinciden con las cifras del Cuadro 4, ya que si existe colaboración interinstitucional (es decir, dos o más dependencias pertenecientes a la misma institución), entonces la sumatoria se modifica.

El Cuadro 4 enlista 26 dependencias pertenecientes a la UASLP, en forma descendente, desde las que más aportan: Instituto de Física (IF) y la Facultad de Medicina (Famed), con más de mil participaciones. En la siguiente columna se presenta el periodo en que se registra el año de la primera y última publicación (según nuestra base de datos), seguida por la columna del año de fundación de la dependencia. Este último dato le da un contexto a las participaciones, pues se observa que, en el caso de las dependencias de la UASLP, hay una brecha entre el año de origen reconocido por las dependencias y cuando se identifica su integración al sistema de comunicación científica vigente, de hecho, se ubica claramente la década de los ochenta como el inicio de la visibilidad en fuentes internacionales de la institución más productiva de SLP; siendo muy similar el comportamiento del representante del sector salud.

El caso del IPICYT es diferente, tanto en su concepción como en su desarrollo, pues obedece a la conjugación de políticas científicas, nacionales de interacción con una promoción y desarrollo local para el cultivo de la ciencia y la aplicación del conocimiento, en el que se reúnen estratégica- 
objetivos (IPICYT, 2007); por lo que la producción de literatura científica es una consecuencia inmediata de la actividad de la institución.

La forma descendente de las participaciones de las dependencias en el Cuadro 4 posiciona a las disciplinas representativas, como las que encabezan los intereses de la actividad científica en SLP, siendo éstas las ciencias físicas (incluye la perspectiva del Departamento de Materiales Avanzados del IPICYT), y la Biomedicina, con un claro eco en la visión de las potencialidades de innovación de la entidad, para Moreno et al. (2008) las tres áreas más prometedoras son la automotriz, nanotecnología y materiales avanzados y el sector salud. .

De la misma forma que el detalle de las dependencias institucionales proporciona un panorama específico de la actividad científica del estado, la división sectorial en la Figura 2 aporta elementos para la caracterización del sistema de investigación local.

Figura 2. Distribución porcentual por tipo de instituciones en la producción científica de SLP

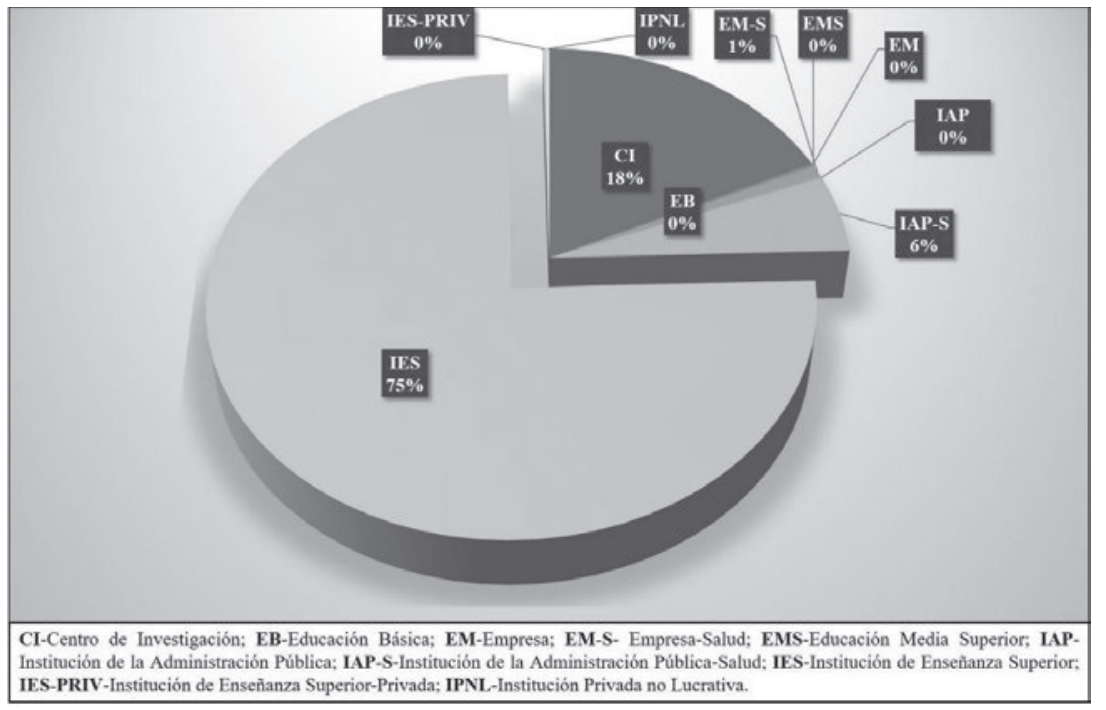

Fuente: elaboración propia.

Para Moreno et al. (2008), el sistema de investigación del estado de SLP se compone de 62 instituciones de educación superior y 6 centros de investigación; mientras que Indra Busines Consultin (Conacyt, 2014: 51) identifica 64 instituciones de educación superior, 7 centros de investigación, 3 oficinas de transferencia tecnológica, 1 centro articulador, 144 empresas Reniecyt, 2 
organismos intermedios, 1 parque tecnológico, 5 redes temáticas, 6 incubadoras y 2 clústers en formación; es decir, la infraestructura que se manifiesta a través de las publicaciones científicas lo que podríamos nombrar la red de instituciones que construyen los investigadores, es más reducida a la que se obtiene cuando se consultan otras fuentes oficiales, o investigaciones con intenciones diferentes a la de la medición de la información, mas no por ello menos informativa y más indicativa en cuanto a la aportación científica de esas instituciones.

En el sistema científico en México predomina la participación de las instituciones de educación superior (FCCT, 2011), y como se observa en la Figura 2, el caso del sistema local de SLP reproduce esta tendencia contundentemente; seguido en porcentajes por los centros de investigación. Cabe destacar aquí la participación de miembros del sector de educación básica e instituciones privadas no lucrativas, que si bien aportan menos del 1\%, su presencia evidencia los procesos de colaboración con instituciones de mayor presencia porcentual y, en consecuencia, de un involucramiento diverso de actores en la actividad científica del estado.

\section{Conclusiones}

Para el sistema de investigación local de SLP se identificaron tres momentos en el estudio de sesenta años de producción científica: uno de inicio (19201979), en el que predominan los temas locales y la publicación individual; el de cimentación (1980-1999), que muestra un incremento constante de la producción científica, la construcción de un marco legal y una red de colaboraciones principalmente en las ciencias físicas.

Por último, está el periodo de proceso de consolidación (2000-2015), en el cual hay un mayor involucramiento de los actores de las redes de colaboración, un importante incremento en la participación de la ciencia de corriente principal y una correlación entre las áreas con potencial de innovación y las disciplinas cultivadas en las instituciones más visibles.

El enfoque de amplio espectro fue posible lograr a través de la armonización de las diferentes fuentes bibliográficas consultadas, el cual deja en claro que la estructura del sistema potosino de investigación muestra una diversidad e inclusión del tipo de instituciones que la conforman y, sobre todo, que SLP no tiene una actividad científica aislada del resto de la comunidad nacional e internacional, por lo que una permanente vigilancia de su producción proporcionaría elementos para una política científica certera con vías a una consolidación y mejor aprovechamiento de sus recursos científicos. 


\section{REFERENCIAS}

Archuby, C. O. 2006. "Aprendiendo matemática con Bradford: propuesta metodológica de enseñanza de uso de los elementos básicos de la matemática en Bibliotecología y Ciencia de la Información [BYCI]", Actas de encuentro. VII Encuentro Asociación de Educadores e Investigadores de Bibliotecología, Archivología, Ciencias de la Información y Documentación de Iberoamérica y el Caribe, 2006. Marília, Brasil: 22-24 de noviembre: 470-486.

Bornmann, L. y L. Leydesdorff. "Which cities produce more excellent papers than can be expected? A new mapping approach, using Google Maps, based on statistical significance testing", Journal of the American Society for Information Science and Technology, vol. 62, no. 10 (2011): 1954-1962.

CERN. 2007. NA62 Detector, en <https://na62.web.cern.ch/na62/Home/Home.ht$\mathrm{ml}>$ (último acceso: 15 de diciembre de 2015).

Chaviano, O. G. 2008. "Aplicaciones y perspectivas de los estudios métricos de la información (EMI) en la gestión de información y el conocimiento en las organizaciones", Biblat., en <http://biblat.unam.mx/es/revista/revista-aibda/articulo/ aplicaciones-y-perspectivas-de-los-estudios-metricos-de-la-informacion-emi-en-la-gestion-de-informacion-y-el-conocimiento-en-las-organizaciones> (último acceso: 22 de 08 de 2016).

Collazo-Reyes, F. y M. E. Luna-Morales. 2002. "Física mexicana de partículas elementales: organización, producción científica y crecimiento”, Interciencia 27, no. 7:347-353.

Collazo-Reyes, F., M.E. Luna-Morales, J.M. Pérez-Angón y M.E. Russell. 2010. “Enriching knowledge production patterns of Mexican physics in particles and fields", Scientometrics, vol. 85, no. 3: 791-802.

Collazo-Reyes, F., M. E. Russell y J.M. Luna-Morales. 2004. "Publication and citation patterns of the Mexican contribution to a 'Big Science' discipline: Elementary particle physics”, Scientometrics, vol. 60, no. 2: 131-143.

Consejo Nacional de Ciencia y Tecnología (Conacyt). 2014. Agenda de innovación de San Luis Potosí. México: Conacyt.

Crane, Diana. 1972. Invisible Colleges; Diffusion of Knowledge in Scientific Communities. Chicago: University of Chicago Press.

Elsevier. 2016. Elsevier Scopus Content., en <https://www.elsevier.com/solutions/ scopus/content $>$ (último acceso: 24 de agosto de 2016).

European Organization for Nuclear Research. 2008-2014. CMS Collaboration., en $<$ http://cms.web.cern.ch/content/cms-collaboration> (último acceso: 15 de noviembre de 2015).

Foro Consultivo Científico y Tecnológico, A.C. (FCCT). 2014. Ranking nacional de Ciencia, Tecnología e Innovación. México: FCCYT.

Foro Consultivo Científico y Tecnológico, AC. (FCCT). 2011. Ranking de producción científica mexicana. México: FCCT.

Garvey, W. D. y B.C. Griffith. 1972. "Communication and information processing within scientific disciplines: Empirical findings for psychology", Information storage and retrieval, vol. 8, no. 3: 123-136. 
Gorbea Portal, S. 2005. Modelo teórico para el estudio métrico de la información documental. Gijón: Trea.

Gorbea Portal, S. , 1996. El modelo matemático de Bradford: su aplicación a las revistas latinoamericanas de las Ciencias bibliotecológica y de la información. México: Centro Universitario de Investigaciones Bibliotecológicas, unam (Monografías, 21).

Hernández García, Y., Dray M. Kleichey J. M. y Russell. 2013. "Enfoques metodológicos para identificar y caracterizar la investigación mexicana en química en bases de datos bibliográficas”, Investigación bibliotecológica, vol. 27, no 59:35-66.

Hurd, J. M. 1996. "Models of scientific communication systems", en , de S. Y. Crawford, J. M. Hurd y A.C. Weller, eds., From print to Electronic. The transformation of scientific communication Medford, NJ: American Society for Information Science, 9-33.

Instituto Potosino de Investigación Científica y Tecnológica (IPICYT). 2007. Anuario 2007. San Luis Potosí: IPICYT.

Kostoff, R. N., J. A. del Rio, J.A. Humenik, E. O. Garcia y A.M. Ramirez. 2001. "Citation mining: Integrating text mining and bibliometrics for research user profiling", Journal of the American Society for Information Science and Technology, vol. 52, no. 13: 1148-1156.

Kretschmer, Hildrun. 1994. "Coauthorship networks of invisible colleges and institutionalized communities", Scientometrics, vol. 30, no. 1:363-369.

Lawani, S.M. 1981. "Bibliometrics-Its theoretical foundations, methods and applications”, LIBRI, vol. 31, no. 4:294-315.

Lawani, S.M. 1986. "Some bibliometric correlates of quality in scientific research", Scientometrics, vol. 9, no. 1-2:13-25.

Lievrouw, Leah A. 1989. "The Invisible College Reconsidered: Bibliometrics and the Development of Scientific Communication Theory", Communication Research, vol. 16, no. 5: 615-628.

Luna-Morales, E., J. M. Russell y C. Mireles-Cárdenas. 2012. "Evolución e impacto de la investigación en la Universidad Autónoma de San Luis Potosí, México: Patrones de publicación y Sistema Nacional de Investigadores", Investigación Bibliotecológica, vol. 26, no. 58: 175-213.

Moreno Mata, A., D. Ríos Jara B. Medina Rivera y R. Alva Funtes. 2008. "Foro Internacional Sinnco", El sistema de innovación regional de San Luis Potosí. Avances y retos, en <http://www.concyteg.gob.mx/formulario/MT/MT2008/MT2/SESION3/MT2_MORENO_RIOS_ALVA_MEDINA.pdf> (último acceso: 14 de noviembre de 2015).

Mutschke, P. y P. Mayr. 2015. "Science models for search: a study on combining scholarly information retrieval and scientometrics", Scientometrics, vol. 102, no. 3: 2323-2345.

Nicholson, S. 2006. "The basis for bibliomining: Frameworks for bringing together usage-based data mining and bibliometrics through data warehousing in digital library services", Information Processing E Management, vol. 42, no. 3: 785-804.

O'Connor, D.O. y H. Voos. 1981. "Empirical laws, theory construction and bibliometrics", library Trends, vol. 30, no. 1: 9-20.

Ortega Priego, J. 2001. "Análisis de referencias basado en un modelo de espacios vectoriales: la investigación en historia contemporánea en Jaén durante 19901995”, Revista española de Documentación Cientifica, vol. 24, no. 4: 369-381. 
Ram, S., y N. Paliwal. 2014. "Assessment of Bradford Law of scattering to psoriasis literature through bibliometric snapshot”, Desidoc Journal of Library E Information Technology, vol. 34, no. 1: 46-56.

Roemer, R. C. y R. Borchardt. 2012. "From bibliometrics to altmetrics A changing scholarly landscape", College E Research Libraries News, vol. 73, no. 10: 596-600.

Selex. 2006. Selex at Work. 19 de junio, en <http://www-selex.fnal.gov/selex_at_ wk.html> (último acceso: 2 de diciembre de 2015).

Subramanyam, K. 1983. "Bibliometric studies of research collaboration: a review", Journal of Information Science, vol. 6, no. 1:33-38.

Thelwall, M., Haustein, S., Larivière, V. y C.R. Sugimoto. 2013. "Do altmetrics work? Twitter and ten other social web services", PloS One, vol. 8, no. 5: e64841.

Thomson Reuters. 2016. Web of Science, en <http://wokinfo.com/latam> (último acceso: 24 de agosto de 2016).

Wolfram, D. 2015. "The symbiotic relationship between information retrieval and informetrics”, Scientometrics, vol. 102, no. 3: 2201-2214.

\section{Anexos}

1. Nombres desglosados de instituciones y dependencias

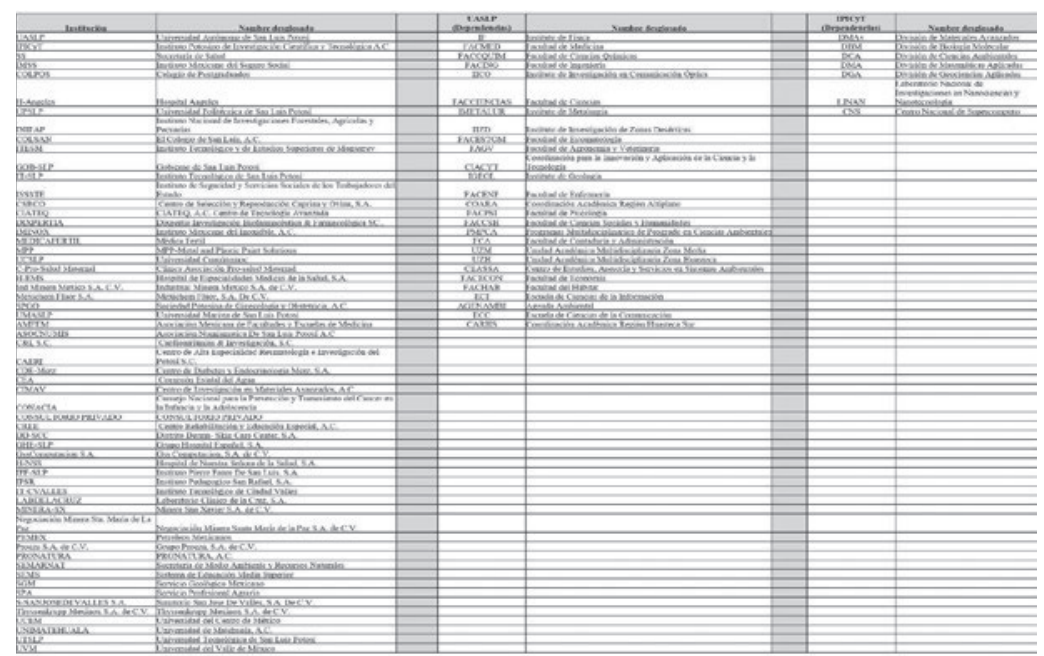


Para citar este texto:

Hernández-García, Yoscelina I.; Navarro-Contreras, Hugo. 2017. "Estudio bibliométrico del sistema de investigación local de San Luis Potosí (1920-2015)". Investigación Bibliotecológica: Archivonomía, Bibliotecología e Información (Número Especial de Bibliometría): 249-270.

http://dx.doi.org/10.22201/iibi.24488321xe.2017.nesp1.57892 